\title{
Future experiments on rare Kaon decays
}

\section{Tommaso Spadaro*i}

Laboratori Nazionali di Frascati dell'INFN

Via E. Fermi, 40, I-00044 Frascati, ITALY

E-mail: tommaso.spadaro@lnf.infn.it

Rare Kaon decays represent a powerful tool for probing possible new physics effects beyond the standard model. In some preminent cases, the information extracted from branching ratio measurement might allow an evidence of new phenomena even in absence of new physics observation at the future Super-B factories. Combined information from different rare Kaon decays might allow to disentangle among different new-physics scenarios. After a brief theoretical introduction, an overview of future experimental activities in this field is given. Near and far future perspectives are discussed.

The Xth Nicola Cabibbo International Conference on Heavy Quarks and Leptons, October 11-15, 2010

Frascati (Rome) Italy

\footnotetext{
*Speaker.

${ }^{\dagger}$ Member of the KLOE and NA62 collaboration.
} 


\begin{tabular}{ccccc}
\hline & $\Gamma_{\text {Shortdistance }} / \Gamma$ & Irreducible theory err. & SM BR & Ref. \\
\hline$K_{L} \rightarrow \pi^{0} v \bar{v}$ & $>99 \%$ & $1 \%$ & $(2.6 \pm 0.6) \times 10^{-11}$ & {$[5]$} \\
\hline$K^{+} \rightarrow \pi^{+} v \bar{v}$ & $88 \%$ & $3 \%$ & $\left(8.2_{-0.7}^{+0.8}\right) \times 10^{-11}$ & {$[5]$} \\
\hline$K_{L} \rightarrow \pi^{0} e^{+} e^{-}$ & $38 \%$ & $15 \%$ & $(3.5 \pm 0.9) \times 10^{-11}$ & {$[6]$} \\
\hline$K_{L} \rightarrow \pi^{0} \mu^{+} \mu^{-}$ & $28 \%$ & $30 \%$ & $(1.4 \pm 0.3) \times 10^{-11}$ & {$[6]$} \\
\hline
\end{tabular}

Table 1: FCNC $K$ modes with relevance for NP tests.

\section{The theoretical landscape}

Kaon is the lightest strange particle, studied since 60's to test fundamental properties of nature. The Standard Model (SM) dynamics at $1 \mathrm{GeV}$ includes remarkably few parameters. The lagrangian,

$$
L_{\mathrm{QCD}}\left(m_{u}=m_{d}, m_{s}\right)+L_{\mathrm{QED}}+L_{\mathrm{IB}}\left(m_{u}-m_{d}\right)+L_{\mathrm{ew}},
$$

depends on light (up and down) and strange quark masses for the QDC part. Isospin-breaking terms arise from QED and from the difference $m_{u}-m_{d}$. The link to physics at the electroweak scale, $L_{\mathrm{ew}}$, breaks many symmetries: parity, $C P$, and flavor. As a consequence, Kaon studies reach the highest sensitivities to $C P T$ - and quantum-mechanics- violation tests. Moreover, lepton-flavor violating $K$ transitions, ultra-rare or forbidden in the SM, have been searched for decades. No signal was found, thus allowing to rule out new physics (NP) with tree (loop) level mediators lighter than $100 \mathrm{TeV}$ $(10 \mathrm{TeV})$ [1]. In recent years, the universality of weak couplings for quarks and leptons have been tested with improved precision by comparing the determination of the Fermi constant from muon decays with that from the combined measurements of nuclei and kaon decays [2]. No deviation is observed from universality, thus ruling out NP models with tree (loop) level mediators lighter than $1 \mathrm{TeV}(10 \mathrm{TeV})$ [3]. At present, the sensitivity in testing NP in lepton-flavor or $C P$ violating transitions with $K$ decays is competitive with that from $B$ decays [4].

In future, the flavor-changing neutral current (FCNC) processes listed in Tab. 1 will be crucial for NP tests. These processes are dominated by $Z$ penguin and box diagrams and do not involve long-distance contributions from processes with intermediate photons. Moreover, hadronic matrix elements can be obtained from BR's of leading $K$ decays, thus allowing precise theoretical predictions. Of uttermost importance, the BR's for $K \rightarrow \pi v \bar{v}$ modes are predicted at few per cent in the SM and can deviate by more than $10 \%$ in many NP models [7]. A simultaneous measurement of the two BR's might allow identification of a specific NP model or, in absence of NP signals, can give direct information on CKM matrix elements. In the remainder of this paper, I will briefly discuss the future experimental activities related to the $K \rightarrow \pi v \bar{v}$ modes.

\section{Searching for $K_{L} \rightarrow \pi^{0} v \bar{v}$}

The essential signature of a $K_{L} \rightarrow \pi^{0} v \bar{v}$ decay is the detection of the two photons from $\pi^{0}$ in absence of any other particle in the final state ${ }^{1}$. The main backgrounds are due to $K_{L} \rightarrow \pi^{0} \pi^{0}$ with

\footnotetext{
${ }^{1} K_{L} \rightarrow \gamma \gamma$ is rejected since the photons are emitted back to back in the $K_{L}$ rest frame, with zero total transverse momentum.
} 
two photons lost and $\pi^{0}$ or $\eta$ production from beam neutron interaction with residual gas in the decay. In order to identify a signal with BR of the order of $10^{-11}$, photon veto must be as hermetic as possible and must include beam exit, while the beam decay region must be of very high vacuum.

The only sharp kinematic constraint is the invariant mass of the two detected photons. This is generally used to reconstruct the decay vertex position. Additional topological constraints are advantageous: a small beam cross section together with the measurement of photon directions allows a redundant evaluation of the vertex position, while a microbunched beam might allow time of flight constraints. The veto system performance and the experiment design are paramount.

The most recent experiment dedicated to $K_{L} \rightarrow \pi v \bar{v}$ search was E391a at KEK [8]. A neutral secondary beam generated from the $12-\mathrm{GeV}$ KEK PS is tranported to a few-meter long, $10^{-7}$ mbar vacuum decay region. The decay volume is enclosed by electromagnetic calorimeters for photon detection. In the forward direction, the calorimeter is made of pure, $7 \times 7-\mathrm{cm}^{2}$ wide, $30-\mathrm{cm}$ deep CsI crystals. The decay region is screened by beryllium and lead filters against neutrons and photons. The $K_{L}$ beam is collimated, with 2-mrad divergence and the beam spot has a width of $4 \mathrm{~cm}$ at the photon veto, thus allowing a geometric constraint for $\pi^{0}$ vertexing. The experiment acquired data in three run periods: Run I in 2004, Run II from february to april 2005 with $1.4 \times 10^{8}$ protons on target (POT), and Run III from november to december 2005 with $1.1 \times 10^{8}$. Signal events are searched in the plane of the $\pi^{0}$ transverse momentum versus the vertex position along the beam. A signal box is defined after after a careful evaluation of the background sources by using a blind analysis (see Fig. 1). No signal events are observed. Combining results from runs II and III E391a collaboration obtains: $\mathrm{BR}\left(K_{L} \rightarrow \pi^{0} v \bar{v}\right)<2.6 \times 10^{-8}$ at $90 \%$ of confidence level [8], improving on previous experiments by a factor of 20 .

E391a can be seen as a first step toward signal identification. The understanding and the experience obtained will be exploited in the future KOTO (also known as E14) experiment [9] at the new J-PARC accelerator [10], presently in preparation. With respect to E391a, $K_{L}$ beam intensity, running time, and signal acceptance are expected to be increased roughly by a factor of 10 each. In the proposed setup, a $30-\mathrm{GeV}$ primary beam with $2 \times 10^{14}$ protons per pulse, will produce an intense $K_{L}$ beam. After a 20 -m long beam line, $K_{L}$ 's will enter a decay region. In the comparison with E391a, not only the beam halo will be suppressed by an additional a factor of 10 with respect to the beam core, but the ratio of neutron to $K_{L}$ in the beam will pass from 40 to 7 . Moreover, the accompanying neutrons will be significantly softer than for the E391a setup, so that it will be easier to reject the background from neutron interactions. The KOTO detector will be an upgraded version of E391: the endcap calorimeter will be replaced by using the $2.5 \times 2.5 \mathrm{~cm}^{2}$ wide, $50-\mathrm{cm}$ deep CsI crystals from the dismantled $\mathrm{KTeV}$ experiment. Better segmentation will lessen the $\gamma-\gamma$ merging probability, while incresing calorimeter depth will lessen the punch-through leakage. Moreover, new veto counters will be installed around beam holes and newly produced high-rate electronics will be used for read-out. Year 2009 was devoted to construction and survey of the $K_{L}$ beamline, while 2010 was dedicated to the construction of CsI calorimeter. An engineering run is foreseen in 2011, while a first physics run with three to seven SM events are expected in a three year run, with an expected single event sensitivity of $\sim 0.8 \times 10^{-11}$. Possible future upgrades are planned to acquire $100 \mathrm{SM}$ events in three years of data taking. 


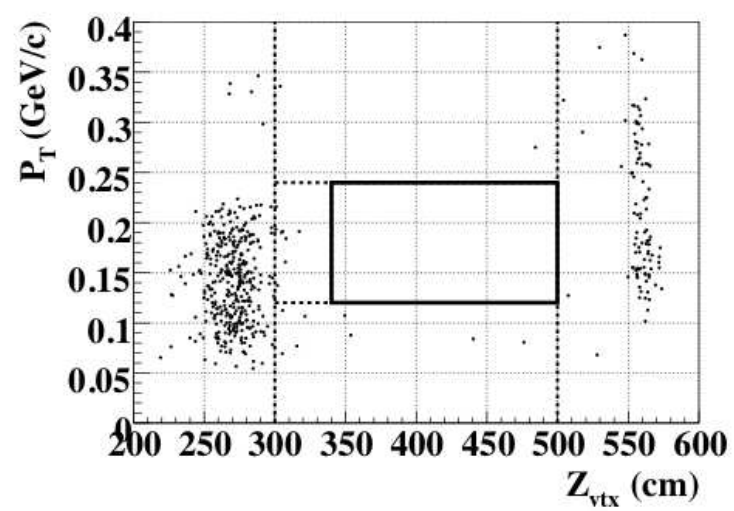

Figure 1: E391a data from Run III. Transverse momentum of the detected $\pi^{0}$ versus $K_{L}$ decay vertex position along the beam. Dots represent data. No event lies in the signal box indicated by the solid line.

\section{Searching for $K^{+} \rightarrow \pi^{+} v \bar{v}$}

The essential signature of a $K^{+} \rightarrow \pi^{+} v \bar{v}$ decay is the detection of a $K^{+} \rightarrow \pi^{+}$decay vertex in absence of any other particle in the final state. The main background foreseen are due to $K^{+} \rightarrow$ $\mu \nu$ with a muon mis-identified as a pion or $K^{+} \rightarrow \pi^{+} \pi^{0}$ with two photons lost. For the first, an excellent and redundant $\pi / \mu$ particle identification is needed. For the second, excellent and hermetic photon vetoing capability is needed. Two-body decays are rejected by cutting on the missing mass at the $K$ vertex, with a rejection on the order of $10^{4}$.

Two different experimental approaches are pursued. In the first, followed by the BNL E787 and E949 experiments at the AGS [11], a $K^{+}$beam is stopped to rest in a sensitive target, emitted pions are tracked in a magnetic spectrometer, and pions are slowed down to rest and the entire $\pi \rightarrow \mu \rightarrow e$ chain is detected. The PID information is redundant and allow a $10^{8}$ rejection. Photon vetoing is demanding in this approach, since photons emitted from a $K^{+}$at rest have low energy. E787/E949 experiments are completed and the final analysis has been finalized in 2008. Seven $K^{+} \rightarrow \pi^{+} v \bar{v}$ events have been identified [11]. The measured BR is compatible with the SM prediction, although with a large uncertainty: $B R=\left(1.73_{-1.05}^{+1.15}\right) \times 10^{-10}$. This result leaves plenty of room for possible NP effects [7].

In the second approach, followed by the NA62 future experiment at CERN SPS [12], highenergy kaons decay in flight. Advanced Cerenkov counters and muon detectors are needed to perform particle identification. The advantage of this aproach compared with stopped- $K$ decay experiments is that photon vetoing has to be performed for high energy photons.

The aim of the NA62 experiment [12] is to detect about $80 K^{+} \rightarrow \pi^{+} v \bar{v}$ events with a $\mathscr{O}(\sim$ $10 \%$ ) signal acceptance and a background on the order of $10 \%$ in two years of data-taking. The design is inspired by years of experience with the NA48 apparatus and infrastructure. For NA62, the K12 beamline at the CERN SPS will be upgraded to increase the intensity by a factor of 50 . In the final setup, a 400-GeV SPS primary proton beam interacts into a beryllium target and produces an unseparated $75-\mathrm{GeV}, 800-\mathrm{MHz}$ beam with $\sim 6 \% \mathrm{~K}^{+}$, corresponding to $\sim 5 \mathrm{MHz}$ kaon decays in a 60-m long fiducial volume. A transverse schematic view of the NA62 detector is shown in Fig. 2.

The guiding principles in the experiment design follow from the need to sustain a high-rate environment while guaranteeing high-resolution timing. The goal is to identify a signal BR of 


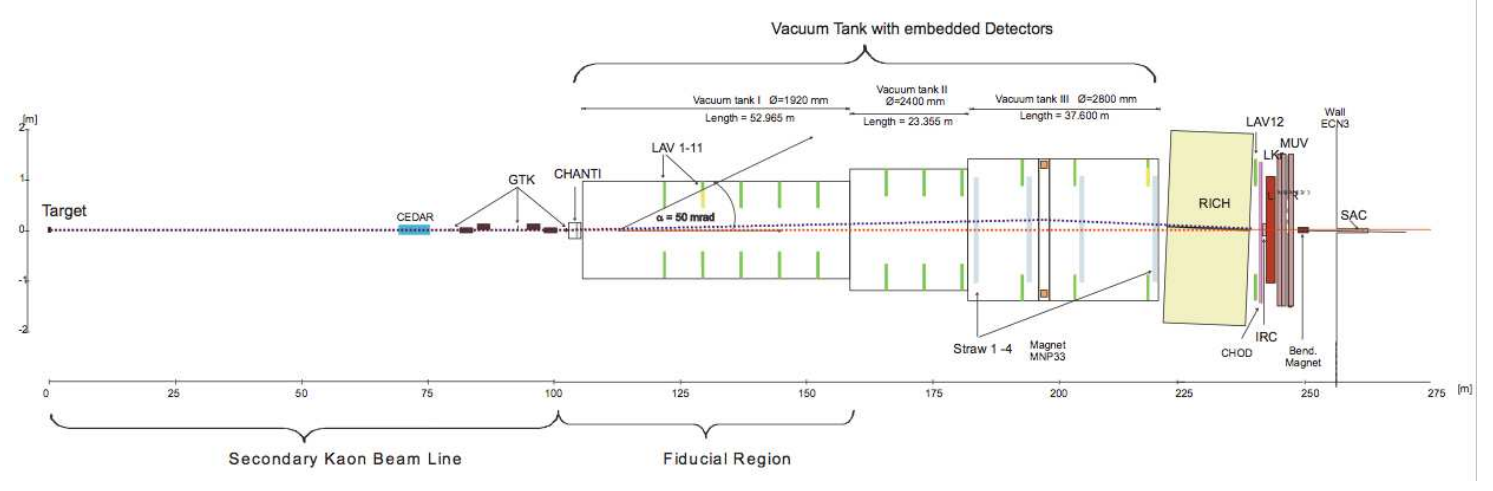

Figure 2: Schematic view of the NA62 detector.

$\sim 10^{-10}$ with a total background rejection of the order of $10^{12}$ against the leading $K^{+}$decay modes.

Two- and three-body decay modes will be reduced by a factor of $\sim 10^{4}$ by cutting on the missing mass of reconstructed candidates. For this purpose, a fast up-stream tracker of every particle in the beam, the so-called Gigatracker [13], is used to measure time of individual beam particles. It will also provide a momentum measurement for single particles with precision much better than that given by the momentum bite of the beam. This system is placed upstream, just before the decay volume, and must sustain rates of up to $800 \mathrm{MHz}$. Downstream to a 60-m long fiducial region for $K$ decays, a straw-chamber magnetic spectrometer is used to measure with high resolution daughter particle momenta.

Further rejection of $K_{\mu 2,3,4}$ and $K_{e 2,3,4}$ background will be obtained with a ring-imaging Cerenkov counter (RICH), used to efficiently and non-destructively identify daughter pions from muons and electrons. The RICH [14], will provide rejection for muons with less than $0.5 \%$ mis-ID probability for events not identified by the muon veto. More than three standard deviations of $\pi / \mu$ separation should be achieved in the $K \rightarrow \pi v \bar{v}$ pion momentum range, $15<p_{\pi}<35 \mathrm{GeV}$. Time determination with a resolution better than $100 \mathrm{ps}$ should be guaranteed, to efficiently match with Gigatracker information. This performance will be obtained bu using a 17-m long, 3-m diameter volume, filled with $1 \mathrm{~atm} \mathrm{Ne}$ gas acting as Cerenkov radiator. In a dedicated test beam for a prototype with $\sim 20 \%$ of the total number of channels, a muon rejection better than $1 \%$ has been measured, with an overall pion loss of few per mil (see fig 3) and a time resolution better than $100 \mathrm{ps}$, these figures holding across the momentum range of interest.

The $\pi / \mu$ separation is critical to achieve sufficient rejection for $K_{\mu 2}$ decays. For this purpose, additional information will be provided by a sampling calorimeter, the so-called muon veto, placed after the $27 X_{0}$ 's of the existing LKr NA48 electromagnetic calorimeter. Rejection of background from nuclear interactions of charged beam particles other than $K^{+}$will be guaranteed by a differential Cerenkov counter, the so-called CEDAR, placed before $K^{+}$'s enter the decay region.

Rejection of modes with $\pi^{0}$ 's and/or (possibly radiative) photons will be provided by a hermetic, high-efficiency photon-veto system, covering from 0-50 mrad $\gamma$ emission angles. This has to provide a rejection factor of $10^{8}$ against $K^{+} \rightarrow \pi^{+} \pi^{0}$. Photons emitted at very small angle, $<\sim 2 \mathrm{mrad}$, will be detected by compact calorimeters in the forward direction, with a required 


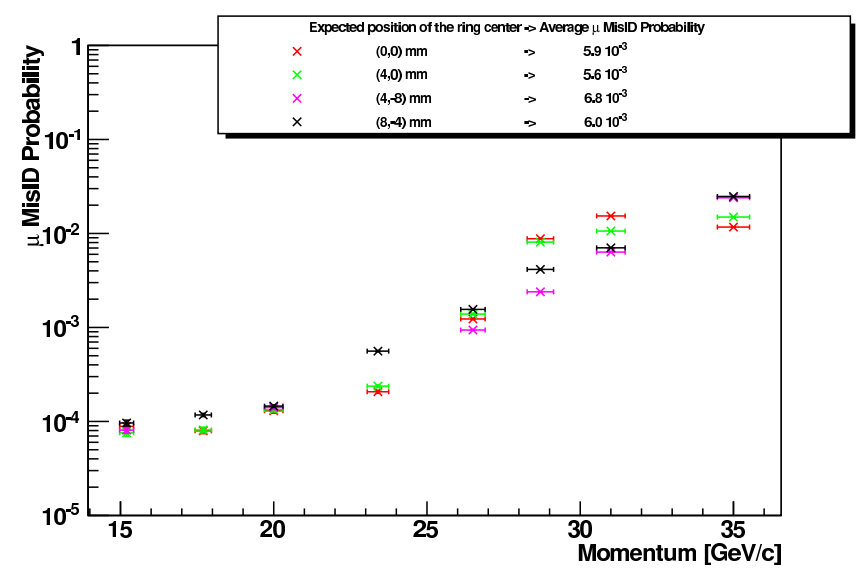

Figure 3: Result from a RICH prototype beam test: muon mis-identification probability as a pion as a function of particle momentum. To estimate the effect induced by the use of a fixed ring position in the prototype, measurements were repeated comparing four alignment positions of the mirror, corresponding to different colors in the plot.

inefficiency of $<10^{-6}$ above $6 \mathrm{GeV}$. In the angular range between $1 \mathrm{mrad}$ and $8 \mathrm{mrad}$, the existing NA48 LKr calorimeter will be re-used, profiting of a measured inefficiency $<10^{-5}$ for photons above $6 \mathrm{GeV}$. At large angle, between $8 \mathrm{mrad}$ and $50 \mathrm{mrad}$, a new system (so-called LAV) will provide $\gamma$ detection with an inefficiency $<\sim 10^{-4}$ above $100 \mathrm{MeV}$.

After an intense R\&D activity, the re-use of SF57 lead glass blocks from the dismounted OPAL barrel electromagnetic calorimeter, already instrumented with R-2238 Hamamatsu phototubes, has been validated for the LAV. The inefficiency measured with dedicated test beams satisfies the requirements and is comparable with other alternatives, including lead/scintillating-fiber or lead/scintillating-tile sampling calorimeters (see Fig. 4, left).
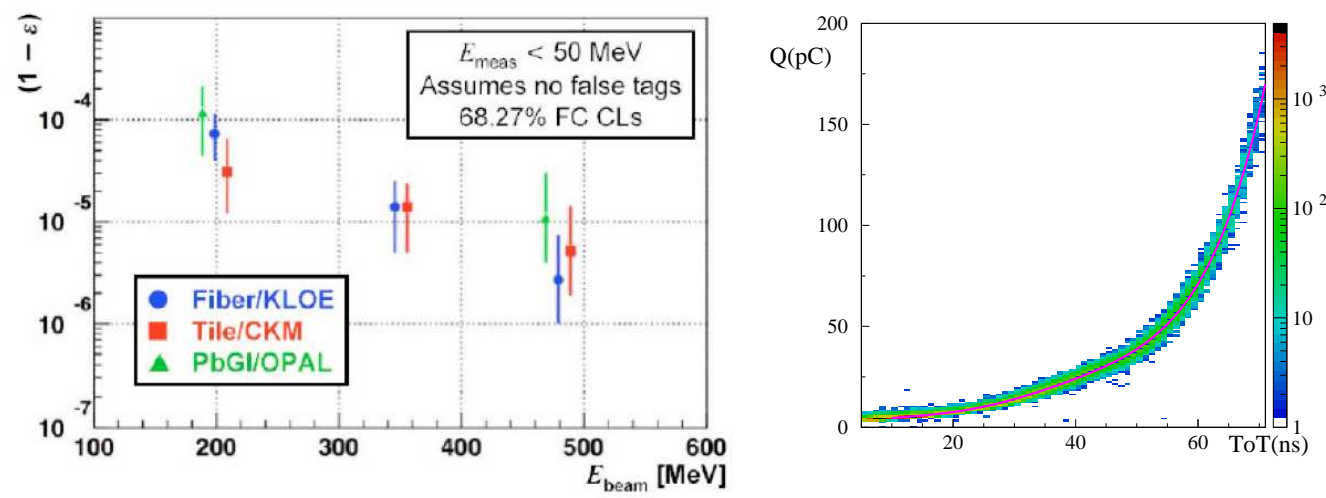

Figure 4: Left: results from large-angle photon veto prototype beam tests. Inefficiency for electron detection as a function of particle momentum is compared for a fiber calorimeter "à-la KLOE" (circles), for a structure alternating scintillator and lead tiles, "à-la CKM" (squares), and for a structure made of OPAL lead-glass blocks (triangles). Right: data from test with electron beam. Charge integrated by a QDC with $0.1 \mathrm{pC}$ LSB versus time over a $30 \mathrm{mV}$ threshold, meausred with a prototype of the LAV front-end electronics and a TDC with 0.25 ps LSB. 
The LAV will be made of 12 stations of increasing diameter to cover hermetically the angular range from 7 to $50 \mathrm{mrad}$. Each station will be composed of four or five layers, staggered to guarantee that incident particles must encounter at least three blocks, corresponding to more than $20 X_{0}$ 's. Since high sensitivity to photons in the range from $20 \mathrm{MeV}$ to $20 \mathrm{GeV}$ is required, the front end electronics must guarantee a wide dynamic range. A simple and cost-effective solution to this problem, easy to scale and to integrate with a common NA62 trigger and data acquisition infrastructure has been adopted: a time-over-threshold discriminator, with multiple adjustable thresholds. Signals will be clamped, split into two, amplificated, and discriminated with two thresholds to allow slewing corrections. The digital output will allow accurate leading and trailing edge time determinations. From the time-over-threshold, a 10\% resolution measurement of the charge will be made (see Fig. 4, right), allowing the LAV system to operate as a calorimeter as well as a veto. Test beam results show that a fractional energy resolution $\sim 10 \% E(\mathrm{GeV})^{-1 / 2}$ and a time resolution $\sim 300 \mathrm{ps} E(\mathrm{GeV})^{-1 / 2}$ are achieved.

In September 2005, the experiment was presented to the CERN SPS Committee and in December 2005 the R\&D was endorsed by the CERN Research Board. In December 2008, the experiment was approved by the CERN Research Board. At present, the collaboration has 191 participants from 25 institutes. Construction and commissioning will last until 2010, and the first physics run is expected to take place in 2013.

\section{References}

[1] L. G. Landsberg, Phys. Atom. Nucl. 68 (2005) 1190-1210 [hep-ph/0410261].

[2] M. Antonelli et al., Eur. Phys. J. C 69 (2010) 399 [arXiv:1005.2323 [hep-ph]] and references therein.

[3] Model independent analysis: V. Cirigliano, J. Jenkins, M. Gonzalez-Alonso, Nucl. Phys. B830 (2010) 95-115. Specific, mostly supersymmetric models: see references 6-9 therein.

[4] See A. Sergi contribution in this conference.

[5] J. Brod, M. Gorbahn, and E. Stamou, arXiv:1009.0947 [hep-ph], and references therein.

[6] F. Mescia, C. Smith and S. Trine, JHEP 08:088, 2006 [hep-ph/0606081].

[7] From the FlaviaNet Kaon working group, http://www.lnf.infn.it/wg/vus/content/Krare_BSM_new.eps

[8] J. K. Ahn et al. [E391a Collaboration], Phys. Rev. D81 (2010) 072004 [arXiv:0911.4789 [hep-ex]].

[9] T. Shimogawa [ J-PARC E14 KOTO Collaboration ], Nucl. Instrum. Meth. A623, 585-587 (2010).

[10] See http://j-parc.jp

[11] A. V. Artamonov et al. [ BNL-E949 Collaboration ], Phys. Rev. D79, 092004 (2009); S. Adler et al., Phys. Rev. D77, 052003 (2008); S. Adler et al., Phys. Rev. D70, 037102 (2004); S. Adler et al., Phys. Lett. B537, 211 (2002).

[12] NA62 Collaboration, CERN NA62 note, NA62-10-07, http: //na62. web. cern. ch/NA62/Documents/NotesDoc/na62-07-10.pdf.

[13] G. Mazza, A. Ceccucci, E. Cortina et al., Nucl. Instrum. Meth. A617, 558-559 (2010).

[14] F. Bucci, G. Collazuol, A. Sergi, Nucl. Instrum. Meth. A623, 327-329 (2010).

[15] A. Antonelli et al., J. Phys. Conf. Ser. 160, 012020 (2009) [Nucl. Phys. Proc. Suppl. 197, 224 (2009)]. 